\title{
Inclusión de la Responsabilidad Social Empresarial en los currículos de Ingeniería Industrial, Administración y Mercadeo en Colombia
}

\author{
Inclusion of Corporate Social Responsibility in the curricula of Industrial Engineering, \\ Administration and Marketing in Colombia
}

Eliasib Naher Rivera Aya

Ingeniero Industrial de la Universidad Distrital y Magíster en Administración de la Universidad Nacional de Colombia. Profesor del Departamento de Ingeniería de la Facultad de Ciencias Naturales e Ingeniería de la Universidad de Bogotá Jorge Tadeo Lozano, Bogotá, Colombia https://orcid.org/0000-0002-5875-1080

eliasib.rivera@utadeo.edu.co

Fecha de recepción: 16 de mayo del 2018 Fecha de aceptación: 16 de julio del 2018

Sugerencia de citation: Rivera Aya, E. N. (2018). Inclusión de la Responsabilidad Social Empresarial en los currículos de Ingeniería Industrial, Administración y Mercadeo en Colombia. Mutis, 8(1), 55-64, doi: http://dx.doi.org/10.21789/22561498.1370

\section{RESUMEN}

En este artículo se presentan los resultados de un estudio orientado a identificar el porcentaje en el que la formación en Responsabilidad Social Empresarial (RSE) se incluye en los planes de estudio de los programas de pregrado en Ingeniería Industrial, Administración y Mercadeo ofrecidos en Colombia al corte de febrero de 2016, y su evolución a partir de información disponible en diciembre de 2012. Al corte de febrero de 2016, se encontró que solamente el $10 \%$ de los planes de estudio de Ingeniería Industrial, el 33\% de los planes de estudio de Administración y el 19\% de los planes de estudio de Mercadeo incluían alguna asignatura obligatoria de Responsabilidad Social Empresarial. Al ampliar el rango e incluir asignaturas que tienen relación directa con la RSE, como Gestión Ambiental y Desarrollo Sostenible, el porcentaje se incrementó al 60\% para Ingeniería Industrial, 55\% para Administración y 32\% para Mercadeo.

Palabras clave: Responsabilidad Social Empresarial (RSE), formación en RSE, inclusión de la RSE en planes de estudio. 


\section{ABSTRACT}

This paper presents the results of a study aimed to identify the percentage in which training in Corporate Social Responsibility (CSR) is included in the curriculum of undergraduate programs in Industrial Engineering, Business Administration, and Marketing offered in Colombia, and its evolution to February 2016, compared to December 2012. Until February 2016, the cut-off date, it was found that only $10 \%$ of Industrial Engineering curriculum, $33 \%$ of Business Management curriculum, and 19\% of Marketing curriculum included a compulsory subject in Corporate Social Responsibility. By extending the range and including subjects directly related to CSR, such as Environmental Management and Sustainable Development, the percentage increased to $60 \%$ for Industrial Engineering, 55\% for Business Management, and 32\% for Marketing.

Keywords: Corporate Social Responsibility (CSR), CSR training, inclusion of CSR in curriculum.

\section{INTRODUCCIÓN}

Los frecuentes escándalos corporativos difundidos por los medios de comunicación, protagonizados a menudo por personas que estudiaron en las facultades de Negocios, Economía, Ingeniería o Derecho, sugieren que algo está fallando en la formación de los gerentes y directivos. No se trata de asignar toda la responsabilidad a las universidades y a sus respectivas facultades, pero dichos escándalos permiten reflexionar sobre cómo inculcar comportamientos éticos y socialmente responsables en los líderes empresariales. Como afirma Kliksberg (2010), la crisis de 2008 surgió de la falta de liderazgo y responsabilidad corporativa. Quienes llevaron a Wall Street al abismo, por su irresponsabilidad, codicia, egoísmo y soberbia, nunca retrocedieron para disculparse por el desastre que causaron, llevando a la ciudadanía a cuestionar: ¿cómo se formaron esos ejecutivos? ¿por qué recibieron un bagaje ético tan precario?, a pesar de recibir una formación técnica de primera línea, ¿qué sucedió con su formación ética?

Persons (2012), Martínez y García (2012), así como Larrán y Andrades (2013), consideran que los escándalos corporativos han impulsado una mayor formación en ética y Responsabilidad Social Empresarial (RSE) en las facultades de Negocios. Esto coincide con la mayor formación en RSE y sostenibilidad que encontraron Moon y Orlitzky (2011) en las escuelas de negocios. No obstante, distintos autores plantean críticas acerca de la formación en RSE y su aplicación. Morgan (1998) considera que, aunque normalmente se piensa que las organizaciones son empresas racionales que persiguen unos objetivos para satisfacer unos intereses comunes, hay evidencias que sugieren que esta visión es más un ideal que una realidad. Las organizaciones se utilizan a menudo como instrumentos de dominación de los intereses egoístas de las élites sobre los demás (p. 263).

Moon y Orlitzky (2011) creen que las facultades de Administración como instituciones no están fomentando la responsabilidad social en la educación de negocios, porque los supuestos de los que parten las teorías, doctrinas y conceptos dominantes del estilo angloamericano de administración orientada a los accionistas son socialmente irresponsables y éticamente dudosos con respecto a la RSE; además, consideran que muchas escuelas de administración se han dedicado a lavar el cerebro de sus estudiantes e infundir la estrecha ideología de los valores de los accionistas, en detrimento de los intereses del resto de la sociedad (p. 329). Por su parte, Laval (2004) afirma que los programas académicos de Administración y de Gestión se ven modificados para ajustarse a las nuevas leyes del mercado, con énfasis en la formación para el trabajo y no para la vida, porque se quiere convertir la escuela en una empresa. En ese mismo sentido, Aktouf (2004) y Chanlat (2002) advierten que el discurso hegemónico ha colocado al dinero y la rentabilidad como criterio principal de la toma de decisiones empresariales, y que las escuelas de gestión han legitimado el enriquecimiento ilimitado de los más ricos.

Debido a estascríticas, la formación en Responsabilidad Social Empresarial está tomando cada vez más fuerza y existe un interés creciente en implementarla en las facultades de Administración, Ingeniería y escuelas de negocios, puesto que: 
(...) son precisamente las universidades, especialmente las facultades de Administración e Ingeniería, las que están en posición de proveer los gerentes e ingenieros de hoy y de mañana. Además de formar gerentes e ingenieros con conocimientos acerca del impacto positivo de una estrategia de RSE para el desempeño a largo plazo de la compañía, las universidades también influencian la percepción que estudiantes y nuevos gerentes tienen de las compañías y sus responsabilidades hacia la sociedad (Sobczak, Debucquet y Havard, 2006, p. 464).

Teniendo en cuenta lo anterior, este estudio buscó identificar el porcentaje en el que la asignatura Responsabilidad Social Empresarial se incluye en los planes de estudio de programas profesionales de pregrado $^{1}$ en Administración, Mercadeo e Ingeniería Industrial en Colombia, al corte de febrero de 2016. Los resultados obtenidos son útiles como línea base para tener un panorama actual y como referente de una mayor inclusión a futuro de la formación en Responsabilidad Social Empresarial en los planes de estudio mencionados.

Al indagar en la literatura consultada sobre alguna clasificación para abordar el tema de la formación en Responsabilidad Social Empresarial, se encontró que Moon y Orlitzky (2011) reconocen tres posibilidades de investigación en la RSE y la sostenibilidad:

1. Estudios comparativos a partir de la inclusión de distintas orientaciones de la RSE, por ejemplo, las cuatro orientaciones propuestas por Garriga y Melé (2004): económica, política, integración social y ética.

2. Investigaciones que examinen los diferentes métodos para establecer la educación en RSE dentro de la corriente dominante, y la efectividad relativa de cada método.

1 En Colombia, la educación primaria consta de cinco años y la educación secundaria, de seis años. Se entiende por pregrado la oferta educativa que ofrecen las instituciones de educación superior, como las universidades, que puede ser de dos años (formación técnica), tres años (formación tecnológica) o entre cuatro y cinco años (formación profesional). En este estudio se revisó la oferta correspondiente a la formación profesional.
3. Investigaciones para medir la efectividad de las diferentes técnicas de enseñanza de la RSE. Algunas preguntas que pueden orientar estas investigaciones son: ¿es el estudio de caso la técnica más efectiva para transmitir un sentido de responsabilidad gerencial a los grupos de interés? o ¿son efectivas actividades en clase como simulaciones de diálogos entre los grupos de interés o stakeholders?

De manera más específica, Setó-Pamies, DomingoVernis y Rabassa-Figueras (2011, p. 606) proponen una clasificación del tema de la formación en Responsabilidad Social Empresarial e indican que la enseñanza de la ética y la RSE se ha examinado desde diferentes perspectivas:

1. Revisión descriptiva de los cursos, su desarrollo histórico y su formato.

2. Recomendaciones pedagógicas para la enseñanza de la ética y la RSE.

3. Juicios analíticos acerca de la efectividad o no de los cursos de ética para modificar valores y actitudes de los estudiantes.

En este caso se contempló la primera perspectiva, dado que se trata de un estudio descriptivo.

\section{METODOLOGÍA}

Para identificar la inclusión de la asignatura Responsabilidad Social Empresarial, o Ética y Responsabilidad Social Empresarial, se realizó un análisis de contenido en internet de tipo exploratorio y descriptivo de la oferta de programas de pregrado en Administración, Mercadeo e Ingeniería Industrial en Colombia, al corte de febrero de 2016. A fin de identificar programas que tuvieran registro calificado vigente y que estuvieran activos, se utilizó la información disponible en el Sistema Nacional de Información de la Educación Superior (SNIES, 2015), el cual fue creado por el Ministerio de Educación para reunir la información relacionada con la educación superior en Colombia.

Para el caso de la oferta de programas de Mercadeo, se encontró un total de 34 programas activos, según el SNIES, los cuales se examinaron; estos incluyen programas de Mercadeo, Mercadología, Marketing y 
Logística, Mercadeo y Publicidad, Administración de Mercadeo, Mercadeo Internacional y Publicidad, y Marketing y Negocios Internacionales. En el caso de los programas de Administración de Empresas ofrecidos, se encontró y se examinó un total de 234 programas activos; estos incluyen programas de Administración, Administración de Empresas y Administración de Negocios. En el caso de los programas de Ingeniería Industrial, se encontró y se examinó un total de 130 programas activos.

Al revisar los planes de estudio, estos se clasificaron en tres niveles. En el primer nivel se incluyeron los planes de estudio que tenían una asignatura obligatoria relacionada con RSE, así como aquellos con una asignatura de Ética, Gestión Ambiental o Desarrollo Sostenible; los planes de estudio clasificados en este nivel debían tener como mínimo una asignatura obligatoria de RSE (Responsabilidad Social Empresarial o Ética y Responsabilidad Social Empresarial). En el segundo nivel se incluyeron los planes de estudio que no tenían ninguna asignatura obligatoria de RSE, pero que tenían como mínimo una asignatura relacionada con Gestión Ambiental o Desarrollo Sostenible, así como algunos que, además de estas, incluían Ética o Ética Profesional. En el tercer nivel se incluyeron los planes de estudio que no tenían ninguna asignatura relacionada con RSE, Gestión Ambiental o Desarrollo Sostenible, pero que tenían como mínimo una asignatura de Ética o Ética Profesional.

En esta medida, los planes de estudio que no se clasificaron en ninguno de los tres niveles permitieron identificar qué porcentaje de los programas de pregrado en Administración, Mercadeo e Ingeniería Industrial no incluían ninguna asignatura obligatoria de RSE, Gestión Ambiental, Desarrollo Sostenible, Ética o Ética Profesional.

Aunque dos de las alternativas para identificar el grado de inclusión de la formación en RSE en los planes de estudio son las encuestas y el análisis de contenido, Rundle-Thiele y Wymer (2010, p. 12) dan una razón que justifica el uso del análisis de contenido en lugar de las encuestas al afirmar que, cuando se usan encuestas, los estudios tienden a arrojar como resultado una mayor inclusión de la asignatura RSE o afines que cuando se utiliza el análisis de contenido, porque los decanos o directivos encuestados pueden sobreestimar la inclusión de asignaturas de RSE en sus facultades o centros. Por el contrario, Rundle-Thiele y Wymer consideran que los trabajos que utilizan el análisis de contenido de los planes de estudio como metodología de investigación pueden ofrecer una visión más aproximada y real de la inclusión de asignaturas relacionadas con la RSE, dado que este método se basa en la obtención de información objetiva y públicamente disponible.

Los resultados de este estudio se compararon con un trabajo previo de Rivera (2013), quien realizó una observación de la inclusión de la RSE en programas de Mercadeo, Administración e Ingeniería Industrial en Colombia al corte de diciembre de 2012, a partir de una muestra con nivel de confianza del $95 \%$ y margen de error del 5\%. Dada la diferencia en la metodología (observación de una muestra versus observación del universo de programas activos), dicha comparación puede tener limitaciones en su precisión debido al $5 \%$ de margen de error de uno de los estudios comparados; sin embargo, permite identificar algunas tendencias de comportamiento relacionado con la inclusión de la formación en Responsabilidad Social Empresarial en los planes de estudio de los programas revisados.

\section{ESTUDIOS PREVIOS}

Con respecto a la aplicación de prácticas de RSE, Lindgreen, Córdoba, Maon y Mendoza (2010, p. 237) encontraron diferencias entre Europa, Estados Unidos y Colombia. En Colombia, las prácticas se basan en mitigar problemas locales a través de estrategias sociales como ofrecer a la población de comunidades muy pobres productos a bajo precio y hacer alianzas con organizaciones para suplir necesidades relacionadas con educación, salud y acceso a microcréditos. También encontraron que una estrategia común para atender las necesidades de los empleados se enfocaba en dos áreas: educación básica para sus familiares y apoyo para la creación de microempresas. Este tipo de prácticas surgen debido a problemas como inequidad, exclusión, paternalismo, corrupción y pobreza, por lo que se proponen acciones de RSE orientadas a mitigar problemas locales a través de estrategias sociales.

En cuanto a la inclusión de la RSE en los planes de estudio, Rivera (2013) presenta algunas cifras. Primero, calculó el tamaño de la muestra utilizando la 
fórmula de la muestra de población finita y, así, estimó el número de planes de estudio a revisar a partir de la población total de tres programas de pregrado: Administración, Mercadeo e Ingeniería Industrial. Entonces, encontró que el $19 \%$ de los planes de estudio de Administración, el $13 \%$ de los planes de estudio de Mercadeo y el $9 \%$ de los planes de estudio de Ingeniería Industrial incluían, al corte de diciembre de 2012, al menos una asignatura de RSE. Al ampliar el rango e incluir el porcentaje de asignaturas que tienen relación directa con la RSE, como Gestión Ambiental y Desarrollo Sostenible, el porcentaje acumulado se incrementó al 45\% para Administración, 26\% para Mercadeo y $60 \%$ para Ingeniería Industrial. Luego, incluyó aquellos planes de estudio que, si bien no tenían una asignatura obligatoria de RSE, Gestión Ambiental o Desarrollo Sostenible, tenían Ética o Ética Profesional, y obtuvo el $33 \%$ de los planes de estudio de Administración, el $32 \%$ de los planes de estudio de Mercadeo y el $24 \%$ de los planes de estudio de Ingeniería Industrial.

De igual manera, existen estudios orientados a identificar la inclusión de la RSE en los planes de estudio de Administración en otros países. En Europa, Matten y Moon (2004) aplicaron una encuesta para analizar la inclusión de la educación en RSE y los niveles en los que se enseña. Con relación a la inclusión de la RSE en los planes de estudio de los programas de pregrado de las escuelas de negocios, encontraron que el $9 \%$ de estas incluía algún curso completo de RSE, el $27 \%$ módulos obligatorios y el $47 \%$ módulos opcionales; el $38 \%$ consideró que incluía el tema de la RSE en otros módulos y cursos. También encontraron que hay una gran diversidad en la comprensión, contextualización y presentación de la enseñanza en RSE, y que solo algunas de las escuelas de negocios toman la iniciativa en esta área, a pesar de que consideran que parte de su función es formar con ética a los futuros gerentes de empresas y líderes. Asimismo, la mayoría de los encuestados reconocieron la necesidad de un mayor compromiso institucional para incluir la enseñanza de la RSE, especialmente mediante la inclusión de este tema en la acreditación de programas y en los sistemas de ranking.

Por su parte, Fernández y Bajo (2010) realizaron un análisis descriptivo de la situación general de la enseñanza de la Responsabilidad Social y Ética Empresarial en las licenciaturas en Administración y Dirección de Empresas ofertadas en 2008/2009, y encontraron que el $27 \%$ ofrecían asignaturas de RSE, siendo el $64 \%$ de estas obligatorias. En un estudio anterior, encontraron que en 2006/2007 el porcentaje era del $21 \%$, lo cual evidencia un ligero incremento en la inclusión de la RSE, que se ha presentado por la necesidad de adaptarse a las exigencias del proceso de Bolonia.

Por otro lado, Setó-Pamies et al. (2011) realizaron un estudio con el propósito de identificar la medida en que se enseñaba RSE en las escuelas de negocios de las universidades españolas. Para ello, utilizaron un análisis de contenido basado en internet de tipo exploratorio y descriptivo. Encontraron que solo el $18 \%$ de las universidades incluían una asignatura específica de RSE en sus currículos.

Asimismo, Moon y Orlitzky (2011) compararon el grado en el que las escuelas de negocios en Norteamérica y en Europa manejan los programas de educación en RSE. A partir de una encuesta realizada en 72 universidades europeas y en 22 universidades norteamericanas, observaron un incremento en la formación en RSE en las escuelas de negocios. Encontraron que la proporción de escuelas de negocios con cursos o módulos obligatorios en el tema de RSE era del 68.3\% para Europa y del $66.7 \%$ para Estados Unidos y Canadá, y que la proporción de escuelas de negocios con módulos opcionales en RSE era del $73.2 \%$ para Europa y del $83.3 \%$ para Estados Unidos y Canadá. Además, encontraron una relación directa entre el prestigio de las escuelas de negocios y su compromiso con la formación en RSE.

Del mismo modo, Larrán y Andrades (2013) encontraron que el $24 \%$ de los programas de pregrado ofrecidos por las universidades españolas en el ámbito de la gestión de organizaciones incorporan en sus planes de estudio asignaturas específicas relacionadas con la RSE, el 32\% incorporan asignaturas específicas relacionadas con la ética y el $23 \%$ incorporan en su itinerario curricular asignaturas relacionadas con la sostenibilidad medioambiental.

Por otra parte, Persons (2012, p. 66) sistematizó su experiencia de incluir los temas de Responsabilidad Social Empresarial y sostenibilidad en un curso de negocios a nivel de pregrado, y recomienda cuatro actividades: revisión y cuestionario sobre el documental The Corporation, y discusión en clase; 
lectura y cuestionario sobre el documento The Evolution of Accountability - Sustainability Reporting for Accountants, y discusión en clase; lectura y cuestionario sobre dos artículos de la revista Fortune (que muestra las compañías más prestigiosas del mundo) y a partir de estos discutir en clase la relación entre la integridad de las compañías, el buen trato a sus empleados y los resultados financieros; y análisis por parte de los estudiantes sobre oportunidades para fomentar la sostenibilidad en su institución universitaria. Adicionalmente, recomienda trabajar en un proyecto para estudiar una empresa y sugerir acciones para aplicar la Responsabilidad Social Empresarial, así como revisar siete casos de estudio donde se analice la ética.

Por último, Nicholls, Hair, Ragland y Schimmel (2013, p. 137) realizaron un estudio orientado a recopilar recomendaciones de cuatro decanos y cuatro jefes de mercadeo sobre cómo incorporar los temas de ética, Responsabilidad Social Empresarial y sostenibilidad en los planes de estudio de los programas de Mercadeo, a nivel de pregrado y posgrado, y la inclusión de estos temas en sus currículos. Encontraron que el tema de ética se incluía en un $51 \%$ de los cursos de Marketing, tanto a nivel de pregrado como de posgrado, el tema de RSE en un $33 \%$ en los cursos de pregrado $y$ en un $50 \%$ en los cursos de posgrado, y el tema de sostenibilidad en un $41 \%$, tanto en los cursos de pregrado como de posgrado.

\section{RESULTADOS}

Al corte de febrero de 2016 , el $19 \%$ de los planes de estudio de los programas de pregrado en Mercadeo en Colombia incluían alguna asignatura obligatoria en Responsabilidad Social Empresarial o Ética y Responsabilidad Social Empresarial. Al ampliar el rango e incluir asignaturas que tienen relación directa con la RSE, como Gestión Ambiental y Desarrollo Sostenible, el porcentaje se incrementó a 32\%. Al contemplar Ética y Ética Profesional, junto con las asignaturas mencionadas, el porcentaje aumentó a $74 \%$.

Con relación a los planes de estudio de los programas de pregrado en Administración en Colombia, al corte de febrero de 2016 se obtuvieron los siguientes resultados: el 33\% incluían alguna asignatura obligatoria en Responsabilidad Social Empresarial
- Ética y Responsabilidad Social Empresarial. Al ampliar el rango e incluir asignaturas que tienen relación directa con la RSE, como Gestión Ambiental y Desarrollo Sostenible, el porcentaje se incrementó a $55 \%$. Al contemplar Ética y Ética Profesional, junto con las asignaturas mencionadas, el porcentaje aumentó a $81 \%$; así que el $19 \%$ de dichos planes de estudio no incluían ninguna asignatura obligatoria que abordara los temas señalados.

En cuanto a los planes de estudio de los programas de pregrado en Ingeniería Industrial en Colombia, al corte de febrero de 2016 se obtuvieron los siguientes resultados: solo el 10\% incluían alguna asignatura obligatoria en Responsabilidad Social Empresarial - Ética y Responsabilidad Social Empresarial. Al ampliar el rango e incluir asignaturas que tienen relación directa con la RSE, como Gestión Ambiental y Desarrollo Sostenible, el porcentaje se incrementó a $60 \%$. Al contemplar Ética y Ética Profesional, junto con las asignaturas mencionadas, el porcentaje aumentó a $84 \%$; así que el $16 \%$ de dichos planes de estudio no incluían ninguna asignatura obligatoria que abordara los temas señalados.

\section{Evolución de la inclusión de la asignatura RSE en los planes de estudio}

En esta sección se presenta la evolución que ha tenido la inclusión de la asignatura RSE en los planes de estudio, mediante la comparación de información correspondiente a diciembre de 2012 y febrero de 2016.

En la Tabla 1 se presentan los porcentajes de inclusión de tres grupos de asignaturas: Responsabilidad Social Empresarial o Ética y Responsabilidad Social Empresarial, Gestión Ambiental y/o Desarrollo Sostenible, y Ética y/o Ética Profesional, en los planes de estudio de Ingeniería Industrial, Administración y Mercadeo correspondientes a diciembre de 2012 (Rivera, 2013) y aquellos correspondientes a febrero de 2016 (información recopilada).

En la Figura 1 se puede observar de manera gráfica la evolución que ha tenido la inclusión de los tres grupos de asignaturas mencionados anteriormente en los planes de estudio de los programas de Ingeniería Industrial, Administración y Mercadeo. 
Tabla 1. Inclusión de la RSE y/o asignaturas afines en los planes de estudio de Ingeniería Industrial, Administración y Mercadeo

\begin{tabular}{ccccccc}
\hline \multirow{2}{*}{ Programas de pregrado } & \multicolumn{2}{c}{$\begin{array}{c}\text { Inclusión de RSE o Ética } \\
\text { y RSE }\end{array}$} & $\begin{array}{c}\text { Inclusión de Gestión } \\
\text { Ambiental y/o Desarrollo } \\
\text { Sostenible }\end{array}$ & $\begin{array}{c}\text { Inclusión de Ética y/o Ética } \\
\text { Profesional }\end{array}$ \\
\cline { 2 - 7 } & dic. 2012 & feb. 2016 & dic. 2012 & feb. 2016 & dic. 2012 & feb. 2016 \\
\hline Ingeniería Industrial (\%) & $9 \%$ & $10 \%$ & $51 \%$ & $50 \%$ & $24 \%$ & $24 \%$ \\
Acumulado (\%) & $9 \%$ & $10 \%$ & $60 \%$ & $60 \%$ & $84 \%$ & $84 \%$ \\
Administración (\%) & $19 \%$ & $33 \%$ & $26 \%$ & $22 \%$ & $33 \%$ & $26 \%$ \\
Acumulado (\%) & $19 \%$ & $33 \%$ & $45 \%$ & $55 \%$ & $78 \%$ & $81 \%$ \\
Mercadeo (\%) & $13 \%$ & $19 \%$ & $13 \%$ & $13 \%$ & $32 \%$ & $42 \%$ \\
Acumulado (\%) & $13 \%$ & $19 \%$ & $26 \%$ & $32 \%$ & $58 \%$ & $74 \%$ \\
\hline
\end{tabular}

Fuente: elaboración propia con base en información disponible en internet.

Figura 1. Evolución de la inclusión de la asignatura RSE en los planes de estudio de Ingeniería Industrial, Administración y Mercadeo entre diciembre de 2012 y febrero de 2016

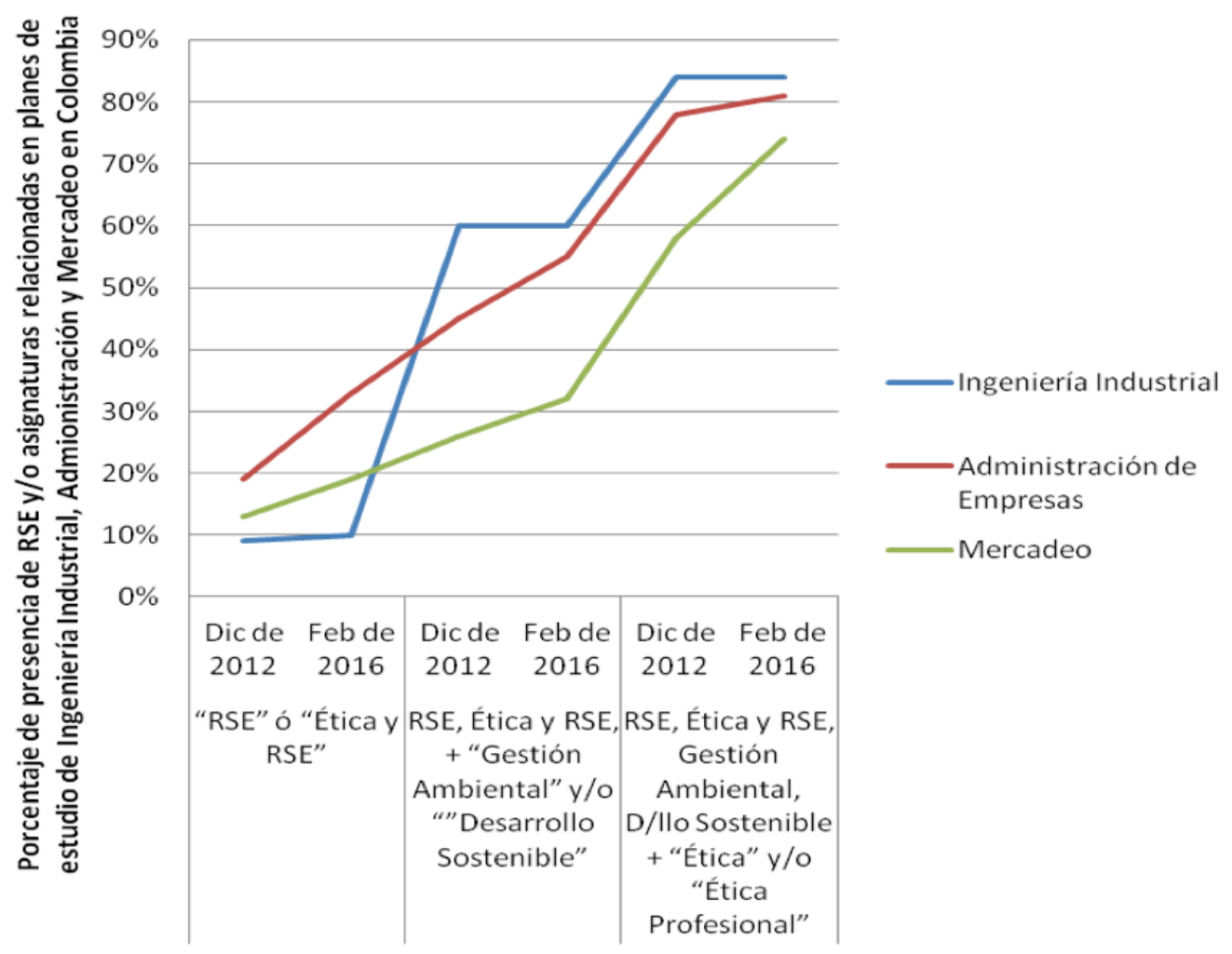

Fuente: elaboración propia con base en información disponible en internet.

\section{DISCUSIÓN}

A pesar de la necesidad de formar profesionales éticos en Administración, Mercadeo e Ingeniería Industrial con responsabilidad social, los resultados obtenidos evidencian que el porcentaje de planes de estudio en estos programas de pregrado que incluían alguna asignatura obligatoria de Responsabilidad Social Empresarial al corte de diciembre de 2012 era muy incipiente (13\% para Mercadeo, $19 \%$ para 
Administración y 9\% para Ingeniería Industrial); no obstante, se observa que, al corte de febrero de 2016, el porcentaje aumentó (19\% para Mercadeo, 33\% para Administración y $10 \%$ para Ingeniería Industrial).

Al ampliar el rango e incluir asignaturas que tienen relación directa con la RSE, como Gestión Ambiental y Desarrollo Sostenible, el porcentaje fue del $26 \%$ para los planes de estudio de Mercadeo, 45\% para Administración y $60 \%$ para Ingeniería Industrial al corte de diciembre de 2012. Al corte de febrero de 2016, los planes de estudio que incluían RSE y asignaturas como Gestión Ambiental y/o Desarrollo Sostenible correspondían al 32\% para Mercadeo, 55\% para Administración y $60 \%$ para Ingeniería Industrial. El cambio del $10 \%$ al $60 \%$ en los planes de estudio de Ingeniería Industrial se debe a una inclusión de la asignatura Gestión Ambiental cercana al $50 \%$ y a la falta de asignaturas de RSE (10\%).

Al corte de diciembre de 2012, los porcentajes acumulados que incluían, además de las asignaturas de RSE, Gestión Ambiental y/o Desarrollo Sostenible, las asignaturas Ética y Ética Profesional fueron del $58 \%$ para los planes de estudio de Mercadeo, $78 \%$ para Administración y $84 \%$ para Ingeniería Industrial. Al corte de febrero de 2016, los planes de estudio que incluían RSE, Gestión Ambiental y/o Desarrollo Sostenible, y Ética y/o Ética Profesional eran del 74\% para Mercadeo, $81 \%$ para Administración y $84 \%$ para Ingeniería Industrial. Por tanto, se evidencia una mayor inclusión en los planes de estudio de Administración y Mercadeo.

Cabe resaltar que, al corte de febrero de 2016, los planes de estudio que no incluían ninguna asignatura de RSE ni asignaturas afines eran del 26\% para Mercadeo, 19\% para Administración y 16\% para Ingeniería Industrial.

\section{LIMITACIONES DEL ESTUDIO}

Dado que la metodología utilizada consistió en un análisis de contenido de información disponible en internet para identificar y registrar las asignaturas de RSE, Gestión Ambiental, Desarrollo Sostenible, Ética y Ética Profesional que se incluían en los planes de estudio de Administración, Mercadeo e Ingeniería Industrial, no se tuvo acceso a información detallada de cada syllabus. Esto representa una limitante para conocer y comparar los objetivos en cuanto a formación, contenidos, metodologías y estrategias de evaluación de cada uno de ellos.

Otra limitante se debe a que en este estudio se identificó la inclusión de la asignatura RSE y otras asignaturas afines mediante la revisión del universo de planes de estudio de programas profesionales en Administración, Mercadeo e Ingeniería Industrial disponibles en el SNIES, para luego comparar los resultados obtenidos con aquellos que presenta Rivera (2013), quien calculó un tamaño de muestra para cada programa utilizando un nivel de confianza del $95 \%$ y un margen de error del $5 \%$. La diferencia entre el uso de una muestra representativa y el uso del universo de los planes de estudio deja abierta la posibilidad de una variación de los resultados debido al margen de error utilizado por Rivera (2013).

\section{CONCLUSIONES}

Aunque la inclusión de la RSE en los planes de estudio de Administración, Mercadeo e Ingeniería Industrial viene aumentando, no deja de ser preocupante que el $26 \%$ de los planes de estudio de Mercadeo, el $19 \%$ de los planes de estudio de Administración y el $16 \%$ de los planes de estudio de Ingeniería Industrial no incluyan la asignatura RSE o asignaturas afines (Gestión Ambiental, Desarrollo Sostenible, Ética o Ética Empresarial). Por un lado, desempeñarse en cargos directivos hace parte del perfil laboral que señalan los planes de estudio de los programas mencionados y, por otro, la sociedad colombiana con frecuencia es testigo de los escándalos protagonizados por gerentes o directores que, mediante la corrupción y la ilegalidad, han demostrado su gran habilidad para acumular riqueza a costa de reducir el beneficio y bienestar de los demás, lo que cuestiona su grado de profesionalismo y ciudadanía.

No obstante, aunque distintos autores (Setó-Pamies et al., 2011; Matten y Moon, 2004) enfatizan en la necesidad de incluir la RSE en los planes de estudio, tanto de Administración como de Ingeniería Industrial, esto no garantiza que los estudiantes vayan a aplicar esos principios en el campo laboral. La universidad no es la única responsable de la formación de buenos ciudadanos, también lo es la familia y la sociedad. Por otra parte, algunos autores (Ruiz Muñoz, 2011; Doane, 2005; Devinney, 2009) consideran que la RSE es más 
una estrategia de manipulación, o un argumento de marketing, y que el interés de las empresas va en contravía del verdadero significado de la RSE. En esta medida, es pertinente contemplar la propuesta de Kletz (2009), quien sugiere realizar investigaciones orientadas a elaborar una pedagogía que conlleve a la implementación de comportamientos responsables (p. 1584).

Teniendo en cuenta que en Colombia hay una inclusión cada vez mayor de asignaturas relacionadas directamente con la RSE, resulta conveniente desarrollar investigaciones que orienten dicha inclusión en los currículos y que presenten recomendaciones curriculares y pedagógicas para la formación en RSE. También puede ser oportuno realizar estudios en los que se identifique de qué manera los empresarios valoran las competencias de los egresados relacionadas con la aplicación de la RSE, ya que con frecuencia se menciona el desbalance entre lo que espera la sociedad y lo que ofrece la universidad, sin que este sea el único criterio para definir los temas y las metodologías en la formación universitaria, pero pensando en que, además de formar buenos ciudadanos, los planes de estudio respondan a las necesidades de las empresas.

\section{REFERENCIAS}

Aktouf, O. (2004). La estrategia del avestruz. Postglobalización, management y racionalidad económica. Santiago de Cali, Colombia: Universidad del Valle.

Chanlat, J. (2002). Ciencias Sociales y Administración. Medellín, Colombia: Fondo Editorial Universidad EAFIT.

Devinney, T. (2009). Is the Socially Responsible Corporation a Myth? The Good, the Bad, and the Ugly of Corporate Social Responsibility. Academy of Management Perspectives, 23(2). doi: 10.5465/ amp.2009.39985540

Doane, D. (2005). The Myth of CSR: The Problem with Assuming That Companies Can Do Well While Also Doing Good is That Markets Don't Really Work That Way. Stanford Social Innovation Review.

Fernández, J. L. y Bajo, A. (2010). The presence of business ethics and CSR in Higher Education curricula for executives: The case of Spain. Journal of Business Ethics Education, 7, 25-38.

Garriga, E. y Melé, D. (2004). Corporate Social Responsibility Theories: Mapping the territory. Journal of Business Ethics, 53(1/2), 51-71.

Kletz, P. (2009). Research in social responsibility: a challenge for management education. Management Decision, 47(10), 1582-1594.

Kliksberg, B. (23 de marzo de 2010). Se necesitan gerentes éticos. Mercados \& Tendencias. Recuperado de http://revistamyt.com/senecesitan-gerentes-eticos/

Larrán, M. y Andrades, F. (2013). Presencia de la responsabilidad social en las titulaciones de grado de las universidades españolas. En Foro de Consejos Sociales de las universidades públicas de Andalucía. Granada, España. Recuperado de http://rsuniversitaria.org/web/images/ stories/Oferta_formativa_en_RS_en_Espana_1. pdf

Laval, C. (2004). La escuela no es una empresa. Barcelona, España: Ediciones Paidós Ibérica, S.A.

Lindgreen, A., Córdoba, J. R., Maon, F. y Mendoza, J. M. (2010). Corporate Social Responsibility in Colombia: Making Sense of Social Strategies. Journal of Business Ethics, 91(S2), 229-242.

Martínez, E. y García, L. (2012). Panorama de la enseñanza en Responsabilidad Social Empresarial en las instituciones de educación superior en México e Iberoamérica. Gestión y Estrategia, (41), 55-69.

Matten, D. y Moon, J. (2004). Corporate Social Responsibility Education in Europe. Journal of Business Ethics, 54(4), 323-337.

Moon, J. y Orlitzky, M. (2011), Corporate Social Responsibility and Sustainability Education: A Trans-Atlantic Comparison. Journal of Management \& Organization, 17(5), 583-603.

Morgan, G. (1998). Imágenes de la Organización. México, D. F., México: AlfaOmega.

Nicholls, J., Hair, J., Ragland, C. y Schimmel, K. (2013). Ethics, Corporate Social Responsibility, and 
Sustainability Education in AACSB Undergraduate and Graduate Marketing Curricula: A Benchmark Study. Journal of Marketing Education, 35(2), 129140.

Persons, O. (2012). Incorporating Corporate Social Responsibility and Sustainability into a Business Course: A Shared Experience. Journal of Education for Business, 87(2), 63-72.

Rivera, E. (octubre de 2013). Presencia de la asignatura "Responsabilidad Social Empresarial" en los planes de estudio de Mercadeo, Administración e Ingeniería Industrial en Colombia. En la XLVIII Asamblea Anual de CLADEA, Río de Janeiro, Brasil. Recuperado de http:// cladea.org/proceedings_2013/wp-content/ uploads/2014/02/2013-XC-0361.pdf

Ruiz Muñoz, M. (2011). Un apunte crítico sobre la Responsabilidad Social Corporativa (RSC/RSE). REDUR, (9), 27-65.

Rundle-Thiele, S. R. y Wymer, W. (2010). Stand-Alone Ethics, Social Responsibility, and Sustainability Course Requirements. Journal of Marketing Education, 32(1), 5-12.

Setó-Pamies, D., Domingo-Vernis, M. y RabassaFigueras, N. (2011). Corporate social responsibility in management education: current status in Spain universities. Journal of Management \& Organization, 17(5), 604-620.

Sistema Nacional de Información de la Educación Superior (2015). Recuperado de http://www.mineducacion.gov.co/ sistemasdeinformacion/1735/w3-article-212396. html

Sobczak, A., Debucquet, G. y Havard, C. (2006). The impact of higher education on students' and Young managers' perception of companies and CSR: an exploratory analysis. Corporate Governance, 6(4), 463-474. 